\title{
Strahlenschutz und Gesundheitskosten
}

Die Eidgenössische Kommission für Strahlenschutz und Überwachung der Radioaktivität (KSR) schätzt die Strahlenschutzsituation in der Schweiz als gut ein (BAG-Bulletin vom 22. August 2005). Beim näheren Durchlesen stösst man auf Forderungen wie «Intensivierung der Ausbildung der Fachpersonen» und «Erfassung der bei Patientinnen und Patienten akkumulierten Dosis». Beide Forderungen sind mit Mehrkosten verbunden, letztere sogar mit Datenschutzproblemen. Mehrkosten sind aus gesundheitsökonomischer Sicht einzig zu verantworten, wenn für die exponierten Patientinnen und Patienten oder die Gesamtpopulation ein relevanter Mehrwert produziert wird. Dieser könnte in Franken pro eingespartes Sievert oder in Franken pro QALY dargestellt werden. Ich wage $\mathrm{zu}$ behaupten, dass im Vergleich dazu jeder monoklonale Antikörper besser abschneidet. Weitere kostenverursachende Vorschriften im Bereiche des Strahlenschutzes liegen ausserhalb des Grenznutzens.
Unter Leitung der Abteilung Strahlenschutz des Bundesamtes für Gesundheit (BAG) soll die Ausbildungsverordnung, namentlich die Ausbildung in dosisintensivem Röntgen und Sachverstand, entschlackt und den wirklichen Bedürfnissen angepasst werden. Die bedürfnisgerechte Ausbildung der medizinischen Praxisassistentin (MPA), wie im Artikel von M. Marchev in der vorliegenden Ausgabe der SÄZ dargelegt, gehört auch in diese geplante Revision der zehnjährigen Strahlenschutzgesetzgebung.

Die Indikation $\mathrm{zu}$ radiologischen Untersuchungen muss im Spital- und Praxisalltag wirklich der aktuellen Evidenz entsprechend gestellt werden. Durch eine restriktivere Indikationsstellung können in relevantem Ausmass Strahlen und Gesundheitskosten gespart werden.

Dr. med. Max Giger, Mitglied Zentralvorstand FMH 


\section{Radioprotection et coûts de la santé}

La Commission fédérale de protection contre les radiations et de surveillance de la radioactivité (CPR) juge que la situation de la radioprotection en Suisse est bonne (Bulletin de l'OFSP du 22 août 2005). Toutefois, en lisant l'article plus attentivement, on relève des phrases telles qu' «intensification de la formation du personnel professionnel» et «enregistrement des doses reçues par le patient». Or, ces deux exigences sont liées à une augmentation des coûts et la dernière pose même des problèmes en matière de protection des données. Sur le plan de l'économie de la santé, des coûts supplémentaires ne sont admissibles que s'ils apportent une plus-value significative aux patients ou à la population en général. Cette valeur ajoutée pourrait s'exprimer en francs par Sievert que l'on évite d'administrer ou en francs par QALY que l'on obtient. J'ose prétendre qu'en comparaison, n'importe quel anticorps monoclonal fournit un meilleur résultat. De nouvelles prescriptions engendrant des coûts supplémentaires dans le domaine de la radioprotection ne présentent qu'une utilité marginale.
Sous la direction de la division de radioprotection de l'Office fédéral de la santé publique (OFSP), la réglementation de la formation, en particulier celle qui concerne la radiologie à fortes doses et la qualification d'expert en radioprotection, doit être simplifiée et adaptée aux besoins réels. Comme le précise l'article de M. Marchev qui paraît dans le présent numéro du BMS, une formation appropriée des assistantes médicales fait également partie de la révision prévue de la législation sur la radioprotection, laquelle date maintenant de 10 ans.

L'indication d'un examen radiologique à l'hôpital ou au cabinet médical doit être posée en fonction des connaissances scientifiques actuelles. Une pose d'indication plus restrictive permettra de limiter notablement l'exposition aux radiations et de réaliser des économies dans le domaine de la santé.

Dr Max Giger, membre du Comité central de la FMH 